Proceedings of the 16th Czech and Slovak Conference on Magnetism, Košice, Slovakia, June 13-17, 2016

\title{
Trapped Field of YBCO Bulk Superconductors Prepared by Infiltration Growth Process
}

\author{
L. VojtKova*, P. Diko And S. PiovarČI \\ Institute of Experimental Physics, SAS, Watsonova 47, 04001 Košice, Slovakia
}

\begin{abstract}
Single grain $\mathrm{Y}_{1} \mathrm{Ba}_{2} \mathrm{Cu}_{3} \mathrm{O}_{7-x}$ (YBCO) bulk superconductors were prepared from different starting precursors. In the first case, $\mathrm{Y}_{2} \mathrm{Ba}_{1} \mathrm{Cu}_{1} \mathrm{O}_{5}$ (Y211) powder as the solid phase and the liquid phase of mixture of $\mathrm{YBa}_{2} \mathrm{Cu}_{3} \mathrm{O}_{7-x}+\mathrm{BaCuO}_{2}+\mathrm{CuO}$ powders were used. In the second case, the solid phase was prepared from the mixture of $\mathrm{Y}_{2} \mathrm{O}_{3}+\mathrm{BaCuO}_{2}$ instead of the conventionally used $\mathrm{Y} 211$, and as the liquid phase a mixture of $\mathrm{Y}_{2} \mathrm{O}_{3}+\mathrm{BaCuO}_{2}+\mathrm{CuO}$ powders was used. The trapped field measurements at $77 \mathrm{~K}$ were performed and microstructure of samples was studied by polarized light microscopy. The positive influence of $\mathrm{Y}_{2} \mathrm{O}_{3}+\mathrm{BaCuO}_{2}$ starting precursor on the oxygenation, Y211 particle refinement and measured trapped field is shown.
\end{abstract}

DOI: 10.12693/APhysPolA.131.1048

PACS/topics: 74.72.-h, 74.81.Bd, 61.50.-f, 74.25.Ha, 81.10.Fq

\section{Introduction}

YBCO bulk superconductors prepared by top-seeded infiltration growth (TSIG) method are perspective materials for practical applications. The main reason for using this method is relatively small volume change of resultant product.

Trapped magnetic field of YBCO superconductors is strongly dependent on their microstructure obtained in the process of single grain production. Desirable microstructure of YBCO superconductors should consist of $\mathrm{Y}_{1} \mathrm{Ba}_{2} \mathrm{Cu}_{3} \mathrm{O}_{7-x}$ (Y123) matrix with small homogeneously distributed $\mathrm{Y} 211$ particles serving as pinning centres. Addition of $\mathrm{CeO}_{2}$ or $\mathrm{BaCeO}_{3}$ is a common way how to refine Y211 particles [1, 2].

Yang et al. [3] published a paper where they proposed a new method for production of GdBCO bulk superconductors by TSIG technique with small 211 particles without Ce addition. They replaced Gd-211 precursor with a mixture of $\mathrm{Gd}_{2} \mathrm{O}_{3}$ and $\mathrm{BaCuO}_{2}$.

In this work, we compared properties of the sample prepared by classic TSIG method with Y211 as a precursor and $\mathrm{CeO}_{2}$ addition with the sample prepared by new TSIG method, where Y211 is replaced with mixture of $\mathrm{Y}_{2} \mathrm{O}_{3}$ and $\mathrm{BaCuO}_{2}$ powders and $\mathrm{CeO}_{2}$ is not added. The preparation of Y211 pellets by this new method should improve properties of YBCO single crystals refining the size of Y211 particles trapped during peritectic solidification and subsequently to enhance trapped magnetic field of these bulk superconductors.

\section{Experimental materials and methods}

Two kinds of samples were prepared by TSIG method. In the first case, the sample was prepared by known method $[4,5]$, where as an initial material of solid phase Y211 powder (Solvay Fluor GmbH, Germany, maximum

*corresponding author; e-mail: vojtkova@saske.sk particle size $\left.d_{\max }=5 \mu \mathrm{m}\right)$ with $0.5 \mathrm{wt} \%$ addition of $\mathrm{CeO}_{2}$ (Aure Remy) was used. Commercially available powders of $\mathrm{YBa}_{2} \mathrm{Cu}_{3} \mathrm{O}_{7-x}$ and $\mathrm{Ba}_{3} \mathrm{Cu}_{5} \mathrm{O}_{8}$ (035, i.e. a mixture of $\mathrm{BaCuO}_{2}$ and $\mathrm{CuO}$ powders in molar ratio of $\mathrm{BaCuO}_{2}: \mathrm{CuO}=3: 2$ ) were weighted in molar ratio of Y123:035 $=1: 1$ and used as liquid source.

In the second case, Y211 phase as a solid state precursor was produced from the mixture of $\mathrm{Y}_{2} \mathrm{O}_{3}+x \mathrm{BaCuO}_{2}$ powders, where $x=1.2$ at.\%, and as a liquid phase the mixture of $\mathrm{Y}_{2} \mathrm{O}_{3}: \mathrm{BaCuO}_{2}: \mathrm{CuO}=1: 10: 6$ powders was used. After pressing into pellet with diameter $20 \mathrm{~mm}$, the mixture was annealed at $910^{\circ} \mathrm{C}$ for $20 \mathrm{~h}$ to form Y211 by reaction: $\mathrm{Y}_{2} \mathrm{O}_{3}+x \mathrm{BaCuO}_{2}=\mathrm{Y} 211$.

The solid phase for both methods was prepared in the form of $12 \mathrm{~g}$ batches uniaxially pressed into pellets with diameter $20 \mathrm{~mm}$. The liquid phase pellets with diameter $32 \mathrm{~mm}$ were pressed from batches of $20 \mathrm{~g}$ in sample A and $16.5 \mathrm{~g}$ in sample $\mathrm{B}$.

The solid phase pellet was placed on the top surface of the liquid source pellet, which, in turn, was placed on the $\mathrm{Yb}_{2} \mathrm{O}_{3}$ plate positioned on $\mathrm{MgO}$ single crystal plate. On the top of the samples $\mathrm{Nd}_{1} \mathrm{Ba}_{2} \mathrm{Cu}_{3} \mathrm{O}_{7-x}$ (Nd123) thin film seeds were placed. The entire arrangement was supported by aluminium oxide plate (Fig. 1a,b).

Both samples (sample A and sample B) for infiltration growth process were heat treated in the same way. First, the samples were heated to $T_{1}=910^{\circ} \mathrm{C}$, and stabilized for $20 \mathrm{~h}$. Then the temperature was increased to the melting point $T=1045^{\circ} \mathrm{C}$ and kept constant for $1 \mathrm{~h}$ to ensure infiltration of liquid phase to solid phase pellet. Cooling process consisted of several steps. After infiltration samples were cooled to temperature $T_{p}=1020^{\circ} \mathrm{C}$ with the rate of $60^{\circ} \mathrm{C} / \mathrm{h}$. From $1020^{\circ} \mathrm{C}$ to $990^{\circ} \mathrm{C}$ they were slowly cooled in three steps for $180 \mathrm{~h}$ (rate $0.3,0.2$, $0.1^{\circ} \mathrm{C} / \mathrm{h}$ ) and then samples were furnace cooled. The time temperature schedule for both samples is presented in Fig. 1c. Prepared bulk crystals were oxygenated in the tubular furnace with oxygen flow atmosphere at temperature $410^{\circ} \mathrm{C}$ for $300 \mathrm{~h}$. 


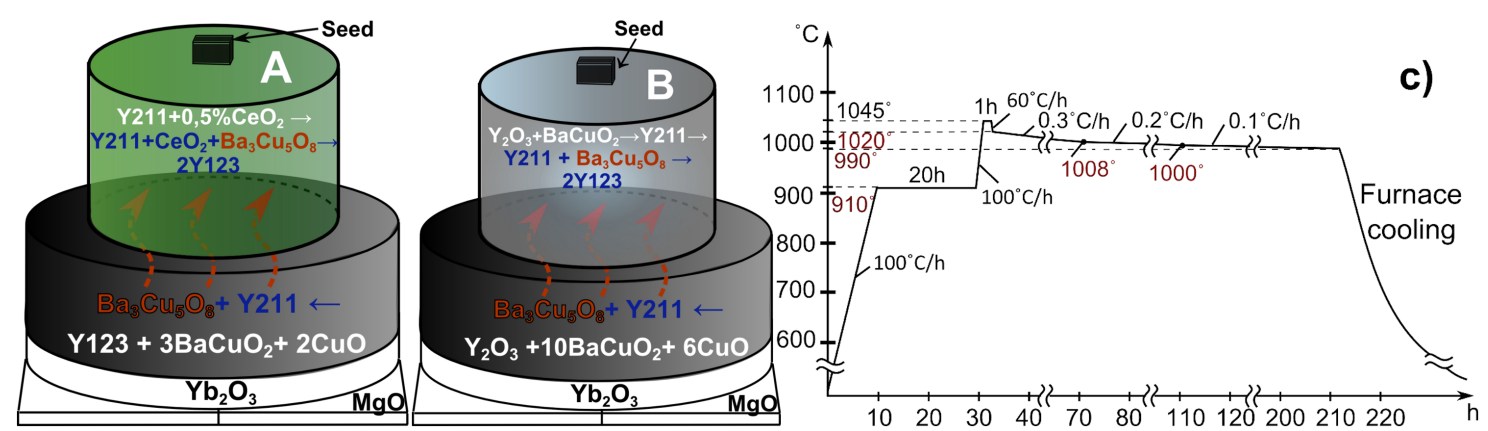

Fig. 1. Schematic illustration of pellet layers of the TSIG process with different starting precursors: (a) sample A method with Y211 solid-phase precursor, (b) sample B - Y211 formed from $\mathrm{Y}_{2} \mathrm{O}_{3}$ and $\mathrm{BaCuO}_{2}$, (c) heat treatment profile used for growth of Y123 bulk single crystal by infiltration growth process.

Microstructure of the samples was studied after polishing by polarised light microscopy and the size of Y211 particles was measured by image processing.

Trapped magnetic field was measured by a home made Hall probe scanner. The samples were cooled in liquid nitrogen and magnetized in an electromagnet with $B=$ $1.4 \mathrm{~T}$ applied parallel to their $c$-axis for $5 \mathrm{~min}$. The distance between sample and pole cone at measurement was less than $1 \mathrm{~mm}$.

\section{Results and discussion \\ 3.1. Results of single crystal growth}

In Fig. 2 the macroscopic images of the top surfaces of samples A and B are demonstrated. The fourfold growth facet lines starting from the seed are clearly visible on the top surface of both samples indicating that the crystal growth was well controlled.
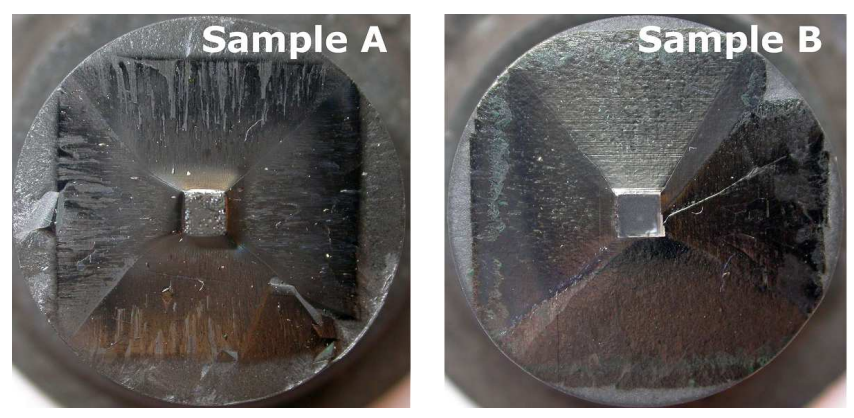

Fig. 2. The top surface of the single-domain YBCO bulk superconductors $(\varnothing 20 \mathrm{~mm})$ with different starting precursors prepared by the TSIG technique.

It is well known that $\mathrm{CeO}_{2}$ addition to samples increases the growth rate of $\mathrm{Y} 123 / \mathrm{Re} 123$ single crystals $[6,7]$. By comparing our samples treated with the same time-temperature regime, it can be seen that the single crystal in sample $\mathrm{B}$ without $\mathrm{CeO}_{2}$ addition is bigger than that in the sample $\mathrm{A}$ with $\mathrm{CeO}_{2}$ addition (Fig. 2). We assume that the main reason is in the difference of Y211 particles size in the samples at peritectic reaction. In the sample A, Y211 powder with average particle size $5 \mu \mathrm{m}$ was used. We suppose that Y211 particles were created by reaction (1) in the sample B and they are smaller than particles in sample A. As the result, the crystal growth in sample B was more intense just due to bigger reaction surface between Y211 and liquid at peritectic reaction

$$
\mathrm{Y}_{2} \mathrm{O}_{3}+\mathrm{BaCuO}_{2} \rightarrow 910^{\circ} \mathrm{C}, 20 \mathrm{~h} \rightarrow \mathrm{Y}_{2} \mathrm{Ba}_{1} \mathrm{Cu}_{1} \mathrm{O}_{5} .
$$

\subsection{Analysis of trapped field}

Figure 3 shows the trapped field profiles of samples A and $\mathrm{B}$ of diameters $20 \mathrm{~mm}$. We can see that sample A has a much lower magnetic trapped field than sample B. In spite of the very low trapped field $B_{\max }=0.013 \mathrm{~T}$, the sample exhibits superconductivity. The lower value of $B_{\max }$ could be caused by worse oxygenation. This has also been confirmed by microstructure analysis, as discussed later (Fig. 4).
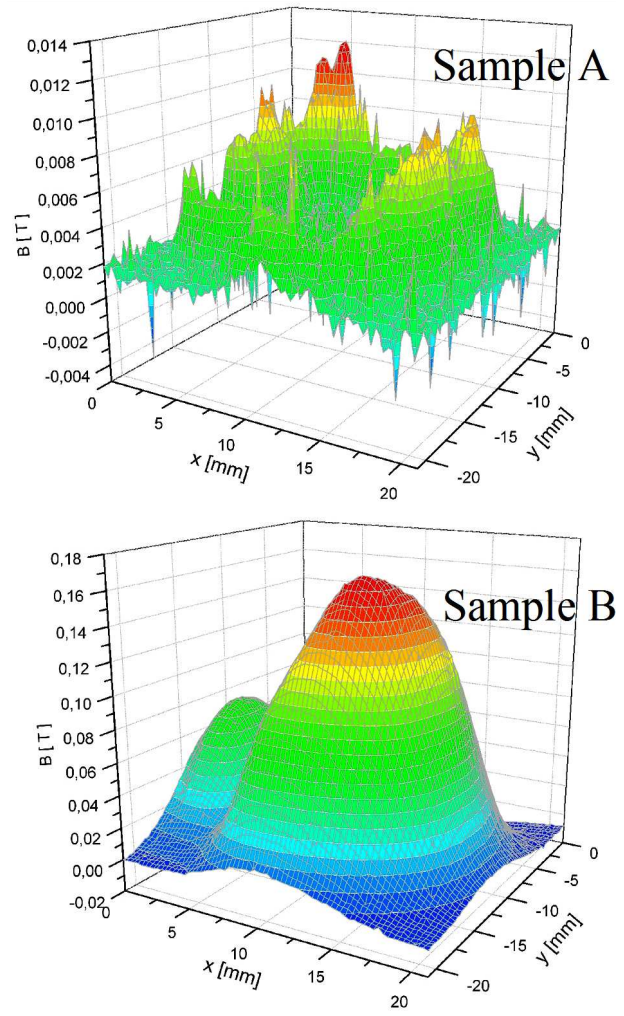

Fig. 3. Trapped magnetic field profiles for the top surface of samples A and B. 
The sample B exhibits typical single-peak trapped field profile on the main part of the sample. Another small peak corresponds to parasitic crystal. The maximum measured trapped field for sample B was $B_{\max }=0.169 \mathrm{~T}$.

\subsection{Structure observation of studied samples}

The macro structures of samples A and B after oxygenation process on the sections parallel to (100) plane are presented in Fig. 4 (left). In both cases the boundaries between $a$ - and $c$-growth fronts (growth sector boundaries GSB [8]) can be seen. The macrostructure of samples is quite different. The structure of sample A is typical for infiltration growth process with small portion of pores (about $13 \mathrm{vol} . \%$ ), which are elongated in horizontal direction. The smaller portion of pores in sample A caused deficient oxygenation. Tetragonal nonsuperconducting Y123 phase visible as horizontal dark areas (Fig. 4, sample A) is present in a high portion.
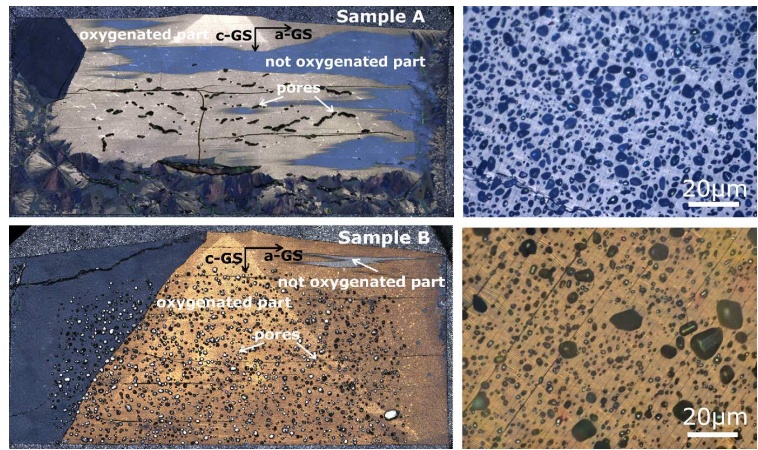

Fig. 4. Cross-sections of samples A and B in polarised light (left). Y211 particles and twins in the sample A and $\mathrm{B}$ (right). Polarised light micrographs taken in the a-growth sector $2 \mathrm{~mm}$ from the seed.

The porosity of sample $\mathrm{B}$ is higher and reaches $22 \mathrm{vol} . \%$. This higher porosity is not typical for samples prepared by infiltration process, but rather for classic top seeded melt growth process (TSMG). In the case of TSMG, it is supposed that the pores are formed from $\mathrm{O}_{2}$ as a result of peritectic reaction at sample melting

$2 \mathrm{YBa}_{2} \mathrm{Cu}_{3} \mathrm{O}_{6} \leftrightarrow \mathrm{Y}_{2} \mathrm{BaCuO}_{5}+\mathrm{Ba}_{3} \mathrm{Cu}_{5} \mathrm{O}_{5.5}+0.75 \mathrm{O}_{2} .(2)$ Probably, the similar effect was realised in the case of sample B. In the preparation process of Y211 precursor, the $\mathrm{Y} 123$ phase could be formed due to excess of $\mathrm{BaCuO}_{2}$, and subsequently at melting by peritectic reaction, $\mathrm{O}_{2}$ pores appear. The pores are helpful in the process of oxygenation of bulk crystal, as it is seen in Fig. 4 (left, sample B). Only a very small part of the sample in the upper right corner is not oxygenized (Fig. 4, left).

In Fig. 4 (right), the microstructure of Y123 bulk crystal with $\mathrm{CeO}_{2}$ addition (sample A) and the microstructure of Y123 bulk crystal produced by new method without $\mathrm{CeO}_{2}$ addition (sample $\mathrm{B}$ ) are shown. The particle sizes were evaluated by image processing software. The fine and spherical Y211 particles are uniformly distributed in the Y123 matrix in both samples. The mean size of most particles in sample $\mathrm{A}$ is in the range of 1.5 to

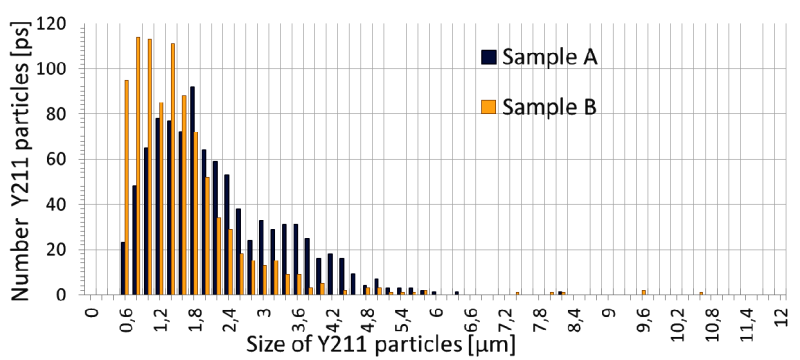

Fig. 5. The size distribution of Y211 particles in the samples A and B.

$5 \mu \mathrm{m}$. There are several large particles in sample B, but mean size of the most particles is in the range of 0.5 to $2 \mu \mathrm{m}$. The size distribution of the Y211 particles in samples is presented in Fig. 5. The smaller Y211 particles in Y123 matrix are more effective in pinning of magnetic flux lines, which positively influences amount of trapped magnetic field.

\section{Conclusions}

In this work, two different methods for Y123 single crystal production by TSIG process were tested. One of them was classic method with Y211 solid phase pellet, in the second method the solid phase was formed from the mixture of $\mathrm{Y}_{2} \mathrm{O}_{3}$ and $\mathrm{BaCuO}_{2}$. We have found that the second method produces YBCO bulks with higher porosity, which significantly improves oxygenation of the sample. Besides that, the second method causes refinement of Y211 particles without addition of cerium oxide. Higher trapped magnetic field measured for the sample prepared by second method was caused by by its much better oxygenation.

\section{Acknowledgments}

This work was realized within the framework of the projects: ITMS 26220120035, ITMS 26220220061, ITMS 26220220041, APVV No. 0330-12, VEGA No. 2/0121/16 and Stefanik Project SK-FR-2013-0025, SAS Centre of Excellence: CFNT MVEP.

\section{References}

[1] P.-W. Chen, I.-G. Chen, S.-Y. Chen, M.-K. Wu, Supercond. Sci. Technol. 24, 085021 (2011).

[2] A. Mahmood, S.D. Park, B.H. Jun, J.S. Youn, Y.H. Han, T.H. Sung, C.J. Kim, Physica C Superconductivity 469, 1165 (2009).

[3] W.M. Yang, X. Zhi, S.L. Chen, M. Wang, J.W. Li, J. Ma, X.X. Chao, Physica C 496, 1 (2014).

[4] G.-Z. Li, W.-M. Yang, Mater. Chem. Phys. 129, 288 (2011).

[5] K. Iida, N. Hari Babu, Y. Shi, D.A. Cardwell, Supercond. Sci. Technol. 18, 1421 (2005).

[6] D. Volochová, P. Diko, V. Antal, M. Radušovská, S. Piovarči, J. Cryst. Growth 356, 75 (2012).

[7] D. Volochová, P. Diko, M. Radušovská, V. Antal, S. Piovarči, K. Zmorajová, M. Šefčiková, J. Cryst. Growth 353, 31 (2012).

[8] P. Diko, Supercond. Sci. Technol. 13, 1202 (2000). 